\title{
LOGROS Y LIMITACIONES \\ EN LA ABSORCIÓN DE LA FUERZA DE TRABAJO EN MÉXICO*
}

\author{
FRANCISCO ALBA
}

El Colegio de México

PARA COMPRENDER LOS ACONTECIMIENTOS QUE actualmente México enfrenta, es necesario remontarse por lo menos al inicio del periodo actual de desarrollo. En la década de los años cuarenta, el pais inicia una época de continuidad y estabilidad económica y política en sus instituciones básicas. El clima político que predomina se caracteriza por un gobierno fuerte y estable, controlado por un partido, el Partido Revolucionario Institucional. El económico se significa por una economía mixta -entre los sectores público y privado- y una economía abierta a la tecnología extranjera, al capital, ideología y formas de vida. La dirección de la estrategia del desarrollo ha estimulado de manera constante la industrialización del país por medio de la sustitución de importaciones. Los resultados no han sido insignificantes: un proceso de crecimiento económico sostenido (el avance del producto bruto supera en promedio al $6 \%$ anual en más de 25 años), coloca al país entre los que han logrado recientemente un alto grado de industrialización. Estos acontecimientos han contribuido a

* Una versión preliminar de este trabajo se presentó en el taller sobre "Crecimiento de población y absorción laboral en el mundo en desarrollo: 19602000", Bellagio, Italia, julio de 1984. 
la formación y consolidación de un consenso social en la estrategia de desarrollo que se desenvuelve desde el inicio de los años cuarenta.

Paralelamente, los anteriores desarrollos, la población del país sufre en este periodo un crecimiento acelerado, y sienta las bases de la ulterior expansión de la fuerza de trabajo. Efectivamente, de los años treinta a los cuarenta, la tasa de crecimiento de la población experimentó un incremento espectacular: de 1.7 a 2.7 por ciento, en promedio anual. Desde ese momento, el crecimiento de la población se mantuvo elevado; durante los años sesenta y el inicio de los setenta llegó a una tasa de 3.4\%. Como resultado de este crecimiento acelerado de la población, la demanda de empleos aumentó a ritmos semejantes.

El presente trabajo intenta explorar la forma en que las instituciones y la estrategia de desarrollo de México han hecho frente al incremento en la fuerza de trabajo. A partir de diversos elementos que se encuentran en la literatura sobre población activa y sobre las estructuras económicas y sociales, se investiga la forma de interacción entre las tendencias económicas, sociales y demográficas y los patrones de absorción del trabajo. En cierta medida, los patrones de absorción aquí descritos se confrontan con las percepciones que por largo tiempo predominaron en la sociedad en cuanto al estado y evolución de la situación laboral del país; punto de gran interés ya que se trata de un ejercicio que seguramente ha generado la adopción de una serie de medidas correctivas respecto a las tendencias de absorción laboral por el aparato productivo.

La parte inicial del trabajo describe el tamaño, la composición sectorial y otros cambios de la población económicamente activa. La central se destina a la discusión de los procesos a través de los cuales se ha dado acomodo a los aumentos de la fuerza de trabajo. La tercera parte se refiere al surgimiento de políticas y programas que recientemente se han concebido para responder de una manera más directa al incremento de la fuerza de trabajo, así como para hacer frente a las condiciones de subocupación generalizada entre amplios sectores de la población. En la parte final se comentan algunos de los dilemas posibles de surgir al cambiar las pautas de absorción de la fuerza de trabajo de propiciarse un viraje en la evolución del desarrollo del país en el corto plazo.

\section{CRECIMIENTO Y CAMBIOS EN LA FUERZA DE TRABA JO}

Inicialmente se proporcionará información sobre la fuerza de trabajo en México. Los datos que se presentan suministran una idea clara de 
la cuantía de los incrementos en ésta, así como de los cambios de estructura de la población activa. Sin embargo, es necesario hacer una advertencia. Los datos y la información en los que se basa este trabajo no siempre son compatibles; hecho que dificulta el efectuar comparaciones sobre la evolución de esta variable en el tiempo. Este escollo tiene que ver no sólo con los problemas de recopilación de las estadísticas, sino con la incidencia de fenómenos coyunturales que en ocasiones podrían distorsionar la interpretación de las tendencias a largo plazo. Por ejemplo, el censo de población que se levantó en México en 1980, durante el periodo del gran auge petrolero, podría encubrir la verdadera naturaleza de las transformaciones de la fuerza de trabajo en el plazo largo, especialmente cuando ese auge fue seguido por una caída drástica de la actividad económica.

La población económicamente activa de México en 1940, asciende, a 6 millones de personas; en 1960 a 11.3 , y en 1980 se incrementa a 22 millones. Estos cambios sustanciales se deben a una elevada tasa de crecimiento de la fuerza de trabajo. En efecto, la tasa de incremento de la oferta potencial de trabajo ya era elevada desde los años cuarenta; en los años cincuenta y sesenta alcanza el $2.6 \%$ anual (Altimir, 1974) y resulta mucho más prominente desde los años setenta. Esta dilatación, se debe al nuevo ingreso de jóvenes mexicanos a la fuerza de trabajo. En términos relativos, la inmigración no ha sido significativa al respecto. Para reforzar lo anterior, es importante observar que los aumentos en la magnitud de la fuerza de trabajo no reflejan una tasa más elevada de actividad entre el total de la población. Incluso, se produce el fenómeno contrario: la tasa de actividad masculina (de 12 años o más) muestra un descenso pronunciado entre 1950 y 1970 , de 88 a 71.8 por ciento. Si bien la tasa de actividad femenina aumenta ligeramente en el mismo periodo, de 13.1 a 16.4 por ciento, el efecto combinado, sin embargo, es un descenso significativo en la tasa total de actividad: de 49.4 a 43.6 por ciento (García, 1975).

Podría afirmarse que, aunque notables, las modificaciones de las tasas de participación por sexo y edad durante este periodo han tenido un impacto relativamente menor sobre el fenómeno de la fuerza de trabajo, si se le compara con el dinamismo de los eventos demográficos - fecundidad elevada y acelerada contracción en la mortalidad- que dan origen a un rápido crecimiento de la población. De hecho, los cambios inmanentes en algunas tasas específicas de actividad, apenas si son perceptibles en la estructura de la oferta laboral, dado el patrón del crecimiento de la población. Por ejemplo, la tasa de actividad económica entre los muy jóvenes, especialmente hom- 
Cuadro 1. México: tasas de actividad por sexo y grupo de edad, $1950-1980(\%)$

\begin{tabular}{|c|c|c|c|c|c|c|c|c|}
\hline \multirow{2}{*}{$\begin{array}{l}\text { Grupo de } \\
\text { edad }\end{array}$} & \multicolumn{2}{|c|}{1950} & \multicolumn{2}{|c|}{1960} & \multicolumn{2}{|c|}{1970} & \multicolumn{2}{|c|}{1980} \\
\hline & $H$ & $M$ & $H$ & $M$ & $H$ & $M$ & $H$ & $M$ \\
\hline $10-14$ & 25.8 & 55.9 & 15.0 & 4.6 & $12.8^{\mathrm{a}}$ & $5.1^{\mathrm{a}}$ & 10.1 & 2.4 \\
\hline $15-19$ & 78.9 & 15.1 & 68.2 & 20.8 & 49.9 & 20.9 & 59.0 & 21.1 \\
\hline $20-24$ & 93.3 & 11.2 & 91.8 & 23.8 & 79.6 & 24.1 & 92.6 & 27.9 \\
\hline $25-29$ & 97.2 & 13.2 & 94.7 & 16.9 & 90.6 & 17.4 & & \\
\hline $30-34$ & 98.4 & 13.3 & 95.9 & 16.0 & 93.2 & 15.7 & & \\
\hline $35-39$ & 98.7 & 13.6 & 96.6 & 17.1 & 94.3 & 15.8 & 97.4 & 18.9 \\
\hline $40-44$ & 98.7 & 13.6 & 96.6 & 18.3 & 93.9 & 16.2 & & \\
\hline $45-49$ & 98.5 & 13.8 & 96.3 & 18.3 & 93.9 & 16.4 & 95.9 & 18.3 \\
\hline $50-54$ & 98.3 & 13.7 & 95.4 & 18.1 & 92.3 & 15.9 & & \\
\hline $55-59$ & 98.1 & 13.8 & 94.0 & 17.7 & 90.6 & 15.1 & 88.9 & 14.9 \\
\hline $60-64$ & 97.8 & 13.5 & 91.9 & 17.0 & 86.1 & 14.1 & & \\
\hline $65-69$ & 97.1 & 13.8 & 88.8 & 15.8 & 81.1 & 12.9 & & \\
\hline $70-74$ & 97.0 & 13.3 & 84.4 & 14.4 & 71.5 & 11.5 & 64.1 & 9.9 \\
\hline+ de 75 & 94.3 & 12.5 & 72.2 & 10.1 & 55.8 & 8.3 & & \\
\hline
\end{tabular}

a Grupo de edad 12-14.

Fuente: Para 1950 (Centro de Estudios Económicos y Demográficos, 1970), con base en el 7o. Censo General de Población; para 1960 (ibidem., 1970), con base en la muestra del 8o. Censo General de Población; para 1970 (García, 1975), con base en el 9o. Censo General de Población; para 1980, estimaciones (PREALC, 1982).

bres, se redujo drásticamente entre 1950 y 1980 : de 25.8 a 10.1 por ciento en el grupo de edad 10-14, y de 79 a 59 por ciento en el grupo 15-19. En cuanto a los varones que tenían 55 años o más, la tasa de participación también descendió de manera significativa: de valores cercanos al 98 por ciento a cerca del 90 (grupo de edad 55-64), y de valores que sobrepasaban el 90 por ciento, a menos del 65 (65 años o más). En contrapartida, las tasas de participación de las mujeres jóvenes aumentaron en el periodo 1950-1980; el cambio más pronunciado se presentó en el grupo de edad 20-24 años, al pasar la tasa de 11 al 28 por ciento. (En el cuadro 1 se observa la evolución de las tasas de participación por edad y sexo.) Sin embargo, la participación femenina en la fuerza de trabajo representó en 1970 solamente una fracción, el $20 \%$ del total de la fuerza de trabajo.

Los cambios demográficos en la magnitud de la fuerza de traba- 
Cuadro 2. México: población activa en la agricultura y fuera de la agricultura, 1940-1980

\begin{tabular}{lllllll}
\hline & \multicolumn{2}{c}{ Agricultura } & & \multicolumn{2}{c}{$\begin{array}{c}\text { Fuera de la } \\
\text { agricultura }\end{array}$} \\
\cline { 2 - 3 } \cline { 5 - 6 } Año & Miles & $\%$ & & Miles & $\%$ & Total \\
\hline 1940 & 3831 & 63.3 & & 224 & 36.7 & 6055 \\
1950 & 4824 & 58.3 & & 3448 & 41.7 & 8272 \\
1960 & 6145 & 54.2 & & 5187 & 45.8 & 11332 \\
1970 & 5293 & 40.9 & & 7662 & 59.1 & 12955 \\
$1980^{2}$ & 7025 & 36.1 & & 12435 & 63.9 & 19460 \\
\hline
\end{tabular}

a Para 1980, estimaciones (Roitman, 1983).

Fuentes: Para 1940-1970, censos de población. La población no clasificada fue asignada a los diversos sectores económicos (Gómez Oliver, 1978: 721).

jo se han visto acompañados por cambios en su estructura. En términos de grandes sectores de actividad económica, todos los sectores de actividad han aumentado el número de personas empleadas, con excepción del sector agrícola, que aparentemente sufrió un descenso en el número absoluto de sus miembros durante los años sesenta (véase cuadro 2). Si bien aún está por confirmarse este suceso, 1 la población que se incorpora a la agricultura, sobre todo después de 1960, lo hace a un ritmo mucho más lento, lo que significa que el grueso de los aumentos de la fuerza de trabajo se incorpora a otros sectores. Durante los años sesenta, los sectores no agrícolas emplearon, por primera vez en la historia del país, un número mayor de trabajadores que los que se ocuparon en la agricultura. La inversión de las proporciones se manifiesta pocos años más tarde, en 1980, cuando el porcentaje de la fuerza de trabajo que se empleó en los sectores no agrícolas representó el $63.9 \%$, casi la misma proporción (63.3\%) que la agricultura empleara en 1940.

1 Se ha argumentado que el censo de 1960 sobreestimó la fuerza de trabajo en la agricultura, y que el censo de 1970 la subestimó. Los problemas de comparabilidad se derivan de lo variable que han sido las fechas de los censos, diversidad de criterios para la clasificación de sectores de actividad, falta de uniformidad al enumerar a los trabajadores familiares no remunerados. Altimir (1974) calculó que la fuerza de trabajo en el sector agrícola era, en 1960, de 5 millones, en vez de 6 millones, basándose en una evaluación de los problemas aquí mencionados y en otros más. Por consiguiente, el total de la población activa del país en 1960 se calculó en 10.2 millones, en vez de los 11.3 millones que proporcionó la información del censo. 
Cuadro 3. México: fuerza de trabajo por sector de actividad, 1950, 1960 y 1970

\begin{tabular}{|c|c|c|c|c|c|c|c|c|}
\hline \multirow[b]{2}{*}{ Sector } & \multicolumn{2}{|c|}{19950} & \multicolumn{2}{|c|}{1960} & \multicolumn{2}{|c|}{$1960^{\mathrm{a}}$} & \multicolumn{2}{|c|}{1970} \\
\hline & Miles & $\%$ & Miles & $\%$ & Miles & $\%$ & Miles & $\%$ \\
\hline $\begin{array}{l}\text { Agricultura } \\
\text { Minería, energía y }\end{array}$ & 4864.9 & 58.3 & 6088.7 & 54.1 & 5048.3 & 49.4 & $5292.7^{b}$ & 40.9 \\
\hline manufacturas & 1237.5 & 14.8 & 1760.3 & 15.6 & 1760.3 & 17.2 & $2829.1^{c}$ & 21.8 \\
\hline Construcción & 263.8 & 3.2 & 414.2 & 3.7 & 414.2 & 4.1 & 609.8 & 4.7 \\
\hline Comercio y finanzas & 732.6 & 8.8 & 1083.4 & 9.6 & 1083.4 & 10.6 & 1397.0 & 10.8 \\
\hline Otros servicios & 1246.4 & 14.9 & 1906.7 & 16.9 & 1906.7 & 18.7 & 2826.5 & 21.8 \\
\hline Total & 8345.2 & 100.0 & 11253.3 & 100.0 & 10212.9 & 100.0 & 12955.1 & 100.0 \\
\hline
\end{tabular}

${ }^{a}$ Estimaciones que corrigen por sobreenumeración en la agricultura (Altimir, 1974)

b En 1970 se excluyen los servicios agrícolas que se prestaron a grupos de unidades agrícolas.

c En 1970 se excluye la parte de distribución de gas que se incluyó bajo "comercio y finanzas"; también se excluyen los servi-

cios de suministro de agua y drenaje que se incluyen bajo "otros servicios" en el sector gobierno.

Fuentes: con base en los Censos de Población (Altimir, 1974). 
El cuadro 3 resume los cambios en la estructura de la fuerza de trabajo. En un intervalo de veinte años, de 1950 a 1970, la distribu. ción de la fuerza de trabajo por sectores ha cambiado de manera significativa. Un sector muy amplio, que incluye mineria, energia y manufacturas, al igual que otro denominado otros servicios, el cual comprende al gobierno, incrementan su importancia a un paso más o menos similar. De poco menos del 15 por ciento pasan a casi el 22. E1 sector de la "construcción" y el de comercio y finanzas también muestran un aumento sostenido tanto en valor absoluto como en peso relativo. En cambio, el sector agrícola, pierde más de 17 puntos porcentuales durante este periodo de veinte años. Del $58.3 \%$ en 1950 , pasa al $40.9 \%$ en 1970 .

Estos cambios en los sectores de actividad económica reflejan un desplazamiento de ocupaciones y empleos rurales-agrícolas hacia puestos de trabajo industriales-urbanos. Algunos de éstos últimos ampliaron muy rápidamente su importancia relativa entre 1950 y 1970. Por ejemplo, según PREALC (1982, cuadro I-61) el grupo de profesionales incrementó su representación de 2.3 a 5.4 por ciento, el de administradores de 0.7 a 2.3 por ciento, y el de oficinistas de 4.3 a 7.2 por ciento. Consecuentemente, esta tendencia se ve acompañada por un ensanchamiento sostenido en la proporción de trabajo asalariado entre la fuerza de trabajo. Según PREALC (1982, cuadro I-62), la proporción aumenta de $51.1 \%$ en 1950 a $63.4 \%$ en 1970 . Por su lado Altimir (1974) calcula que el trabajo asalariado se eleva de $53 \%$ en 1950 a $66.5 \%$ en 1970 . Sin embargo, los trabajadores independientes, así como los empresarios, aún representan cerca de una tercera parte de la fuerza de trabajo remunerada (los trabajadores familiares no remunerados fueron excluidos del cómputo).

El cuadro informativo sobre la desocupación y subocupación de la fuerza de trabajo en México se refiere a fechas más recientes. Según el censo de población de 1960 , únicamente el $1 \%$ de la población se encontraba desocupada (100 000 personas). En 1970, diez años después, esta cifra asciende al 3.8\% (500 000 personas). Sin embargo, con base en datos de encuestas levantadas en áreas metropolitanas (las tres mayores del país), la cifra del desempleo se elevó para ese mismo año al 7\%. La tasa de desocupación "urbana" permaneció en ese nivel durante los años setenta. En 1977 se estimó en $7.6 \%$. A partir de ese momento disminuyó de manera gradual, pero constante, entre $1978(6.9 \%)$ y el inicio de $1982(3.7 \%)$. Sin embargo, al final de 1982 y durante 1983 alcanzó, por lo menos, el 8 por ciento.

Las cifras de desocupación abierta transmiten una visión limitada del mercado de trabajo. No capturan la naturaleza estacional e inter- 
mitente de muchas actividades, la baja productividad de innumerables empleos y el carácter "tradicional" de muchas ocupaciones. La subocupación, como rasgo del mercado laboral, ha sido calculada y aproximada de diversas maneras. Los cálculos del nivel de subocupación, basados en el ingreso percibido, estiman que el subempleo en 1970 afectaba de un 37 a un 45 por ciento de la fuerza de trabajo, al encontrarse que entre 4.5 y 5.8 millones de activos percibian montos muy bajos de ingreso. 2 Sectorialmente, los porcentajes más elevados de subocupación se encontraron en la agrícultura (de 62 a 68 por ciento); los más bajos en energía - petróleo y electricidad--, transporte y gobierno (Grupo de Estudio del Problema del Empleo). Sin embargo, estimaciones de los niveles de subocupación derivadas del criterio sobre números de horas trabajadas arrojaron, también para 1970 , niveles más bajos que van del 14.6\% (Urquidi, 1974) al 16\% (Morelos, 1974) del total de la fuerza de trabajo. Pese a las enormes discrepancias encontradas al aplicar los distintos criterios utilizados para medir la cuantía de la desocupación, resulta claro que las estimaciones revelan la enorme subutilización de la fuerza de trabajo en México.

Es comprensible que los niveles de subocupación y desocupación se encuentren ligados al funcionamiento de la economía y la sociedad, más aún a la forma como el sistema absorbe la fuerza de trabajo. La situación del empleo es sólo un aspecto de una secuela más amplia de condiciones en la absorción del mismo. Es innegable que las fuerzas sociales, económicas y demográficas, así como otras tendencias, influyen en la naturaleza y modalidades que este proceso adquiere. En relación con los problemas de subocupación y desempleo, lo menos que se puede decir es que estas fuerzas y tendencias no marchan en forma balanceada y congruente: algunas de éstas pueden desplazarse muy por encima o muy por debajo de lo requerido, o bien es posible la inadecuación entre ellas. Por ejemplo, el crecimiento de la fuerza de trabajo - la población- puede ser muy acelerado; o bien la acumulación de capital puede no ser lo suficientemente rápida; o los procesos tecnológicos inadecuados, o algunas políticas institucionales y sociales (relacionadas con la legislación laboral, la educación, la capacitación) pueden resultar débiles o contraproducentes en sus efectos sobre el mercado laboral.

2 En mi opinión, no deberían emplearse los criterios sobre el ingreso para calcular los niveles de subocupación. Los salarios bajos se encuentran ligados a las fuerzas del mercado y a instituciones sociales que no coinciden necesariamente con los factores que responden al grado de utilización de la fuerza de trabajo. Desde luego que los niveles de ingreso pueden ser utilizados como un indicador de ocupación parcial, escasa calificación y baja productividad. 
Posteriormente se intentará explorar algunos aspectos de esta interrelación. El presente trabajo busca un sentido que permita enlazar el tema de cómo la fuerza de trabajo ha logrado encontrar un lugar dentro de un determinado ambiente económico y social, con el tema de la forma en que la economía y la sociedad mexicanas han respondido ante una fuerza de trabajo en rápido aumento. ¿Son tal vez dos posibilidades de la misma cuestión? La interpretación que en seguida se desarrolla muestra que éste ha sido un proceso con éxitos y fracasos. El proceso de incorporación de la fuerza de trabajo al sistema social y económico del país, se concibe como una cadena de interacciones entre las fuerzas demográficas, económicas y sociales. De estas acciones y reacciones resulta un patrón de absorción de fuerza de trabajo. Lo que a continuación se presenta es un intento por descubrir algunos aspectos de este patrón, a la luz del estilo de desarrollo que ha caracterizado a México en el pasado reciente.

\section{DEL "MILAGRO" A LA REALIDAD}

Hasta 1940, México contaba con innumerables características que lo hacían una nación predominantemente rural y agrícola. Más de 100000 localidades de aproximadamente 1000 habitantes daban acomodo al $49.8 \%$ (9.8 millones) de la población. Tan sólo el $20 \%$ de ésta vivía en localidades "urbanas" de 15000 habitantes o más. Entre ellas, la ciudad de México con 1.5 millones de habitantes, representaba el $31 \%$ de la población urbana y el $8 \%$ de la población total. En general, las condiciones rurales eran desfavorables, como puede inferirse del hecho de que el producto agrícola era únicamente el $20 \%$ del producto interno total, mientras que la población ocupada en actividades agrícolas representaba casi las dos terceras partes del total de activos (véase cuadro 2). Por este motivo, no es difícil aseverar que el grueso de la población estaba constituido por familias de escasos recursos que se sustentaban de una agricultura "precaria". La economía campesina era el modo de vida predominante.

Frente a esta situación, en el sector agrícola se consolidan dos tipos de políticas: la primera, comprende la distribución de la tierra y la organización ejidal como instituciones ligadas a un sistema político firmemente establecido. La segunda, se refiere al impulso de la agrícultura comercial como contraparte del sistema económico que promueve la modernización e industrialización del país. Si bien estos dos tipos de políticas produjeron en la agricultura una dualidad, al combinarse lograron que el proceso también encerrara una cierta funcionalidad social, lo cual dio origen a un patrón estable de acomodo 
demográfico. El diseño de estas políticas ciertamente no partió de consideraciones demográficas. Pese a ello, se podría pensar que la permanencia y continuidad de éstas no es un desarrollo independiente de la evolución demográfica del país. En la primera se proporcionaron tierras para nuevos asentamientos rurales, $y$ en la segunda se produjeron alimentos para los trabajadores urbanos, y materias primas para la transformación industrial.

El aumento de la tierra ejidal ilustra la importancia de la política agraria en el proceso de otorgar alojamiento al incremento de la población. La superficie ejidal ascendió a 3.5 millones de hectáreas en 1940 y a 12.8 millones en 1970 . Es importante observar, que la tierra privada de cultivo aumentaba a un ritmo similar. En consecuencia, la participación de la tierra ejidal en la totalidad de la tierra cultivable permaneció relativamente estable en este periodo. La proporción descendio del $48 \%$ en 1940 al $45 \%$ en 1960; posteriormente se elevó a $55 \%$ en 1970 (Hewitt de Alcántara, 1980). En general, la tierra bajo cultivo (antigua y nueva) aumentó entre un 4.5 y un 5.5 por ciento anual promedio durante la mayor parte del periodo en cuestión, 1945-1964 (Gómez Oliver, 1978). Este proceso de expansión de la tierra "abrió espacio" a una población rural en crecimiento sostenido. La población activa en la agrícultura se incrementó de manera sustancial entre los años cuarenta y cincuenta, sin embargo, existen discrepancias con relación a la cantidad exacta: el aumento neto de la fuerza de trabajo agrícola fue de por lo menos 1.3 millones de activos, y tal vez un millón más (véanse cuadros 2 y 3 ).

En el presente ensayo se intenta explicar cómo debido a la política de la reforma agraria pudo acomodarse una proporción considerable de los aumentos en el crecimiento de la población rural en las áreas rurales (antiguas y nuevas), lo cual reprodujo y expandió la organización "campesina". En cuanto a la distribución de la tierra, la reforma se convirtió en uno de los mecanismos por medio de los cuales la sociedad mexicana hizo frente a los aumentos de la fuerza de trabajo. Mediante dicho instrumento, el sistema político fue capaz de responder a una demanda constante y sostenida de tierra, lo que a su vez le otorgo estabilidad y legitimidad. Sin embargo, la redistribución de la tierra no fue el único factor de desarrollo que se llevó a cabo en el país. La expansión de la frontera agrícola tuvo lugar también en nuevas tierras de riego. El total de ellas se incrementó de 1 a 4 millones de hectáreas entre 1941 y 1964. La mitad de esta expansión correspondió a los distritos oficiales de riego que aumentaron de un poco más de medio millón de hectáreas a 2.1 millones durante el mismo periodo (Hewitt de Alcántara, 1980). La irrigación dio susten- 
to a una agricultura empresarial dinámica, la cual proporcionó empleo, bajo bases temporarias y permanentes, a un buen número de campesinos y trabajadores agrícolas. De esta manera, el desarrollo de la agricultura moderna también actuó como una estrategia de asentamiento de población.

El desarrollo rural dual anteriormente expuesto puede ser entendido como una institución funcional que acomodó, sin provocar tensiones, parte del aumento de la población rural; asimismo generó excedentes alimentarios para una población urbana en crecimiento. Estas funciones, desde luego, no se llevaron a cabo por medio de patrones aislados: por un lado estaban los asentamientos humanos originados por la organización campesinoejidal, y por otro, la producción de alimentos de las empresas modernas. Ambas organizaciones compartieron las responsabilidades de acomodar y alimentar a la población. La agricultura empresarial, además de generar excedentes, absorbió trabajo contratado; la economía campesina, además de dar acomodo a gran parte de sus miembros, produjo excedentes -granos básicos, legumbres, vegetales, etc.-, para el mercado (Gómez Oliver, 1978). Sin embargo, este proceso también significó la perpetuación de una dualidad caracterizada por un número relativamente pequeño de agricultores frente a una masa de campesinos. Se calcula que de los 2.6 millones de unidades productivas en la agricultura en 1970 , el $86.6 \%$ eran unidades de campesinos, el $1.8 \%$ unidades empresariales y el 11.6\% restante "unidades de transición" (CEPAL, 1982; 111-121). 3

El proceso de expansión de la tierra para cultivo ofreció oportunidades de absorción laboral; éstas, sin embargo, eran sólo algunas opciones entre las existentes. La ciudad también ofrecía alternativas. Con el incremento de la población se elevó el flujo del campo a la ciudad. Se estima que entre 1940 y 1960 , cerca de 1.1 millones de personas activas de las unidades agrícolas de producción se movilizaron hacia los sectores secundario y terciario (Centro de Investigaciones Agrarias, 1974; 315). 4

${ }^{3}$ Una distinta tipología de los grupos sociales en el campo calcula que un $78 \%$ de las unidades productivas --dentro de esta tipología el total de unidades productivas ascendían a 3.2 millones en 1970- son campesinos en descomposición; un $12 \%$ lo conforma un grupo de economía familiar campesina; y el $10 \%$ restante lo constituye el sector empresarial del campo, de ellos el 1.7\% está compuesto por propietarios privados y el $8.3 \%$ por ejidatarios (Luiselli Fernández, 1977:213-217).

4 El cálculo sobre el volumen de los migrantes rurales varía según el uni- 
La política de industrialización, como proyecto de desarrollo del país, favoreció e hizo posible la incorporación de la mano de obra en el medio urbano. En su etapa de planeación, los supuestos de sustentación de la política industrializadora eran que el crecimiento de las ciudades y los avances en la industria significaban fuerzas capaces de absorber el aumento de la población, así como de elevar su nivel de vida. La incipiente industrialización no exigía gran capacitación para el desempeño de las tareas requeridas. Incluso, la fuerza de trabajo rural no parecía tener dificultad para incorporarse al mercado de trabajo nacional (urbano). La creencia general era que el sistema socioeconómico tenía suficiente espacio para dar acomodo a las tendencias demográficas.

La imagen del México actual es precisamente, la imagen del surgimiento y expansión de las clases medias y de los habitantes urbanos. Clases medias, proletariado industrial y burocracia surgen como grupos sociales que desde los años cuarenta capturan visiblemente el crecimiento de la población urbana. El cuadro general que en esos años existe de la economía y sociedad mexicanas está caracterizado por índices socioeconómicos en ascenso. El patrón de desarrollo por industrialización sustitutiva se veía como un camino prometedor que seguramente gestaría el "milagro mexicano". Es posible que gran parte de las cifras que se mencionan sobre la evolución social y económica de esos años son demasiado agregadas, sin embargo, proporcionan una idea clara de los logros alcanzados por el camino que tomó el desarrollo mexicano. Por ejemplo, el porcentaje de familias de escasos recursos descendió entre 1950 y 1963 en términos absolutos y relativos: de 3.1 millones de familias $-60 \%$ - en 1950 a 2.6 millones $-35 \%$ - en 1963 (van Ginneken, 1980:19). Por lo demás, es bien sabido que la mayoría de los pobres se encuentran en áreas rurales.

Pese a que la capacidad de absorción del sistema tuvo sus restricciones y limitaciones, éstas no fueron muy aparentes durante las dos o tres décadas iniciales de este estilo de desarrollo. No toda la población se había asimilado y beneficiado del mismo. Frente a ello, se presuponía y se daba por sentado que eventualmente se produciría la asimilación de la población marginada por la corriente principal, industrial, moderna y en ascenso. Es fácil ver, en retrospectiva, que tanto los sectores informales urbanos como la economía campesina llenaban muchos vacíos que no eran cubiertos por la corriente más importante de desarrollo. Al final de los años sesența y durante todo

verso de la población en cuestión, la definición adoptada de localidades urbanas y rurales, y la inclusión de desplazamientos permanentes y temporales. 
el periodo de los años setenta, los acontecimientos y la investigación mostraron la fuerza y papel de esas instituciones, las cuales, fuera del proyecto de desarrollo, resultaron ser mecanismos de "colchón" en el proceso global de absorción del trabajo.

En todo caso, la evolución del desarrollo que tuvo México sin tropiezos, comenzó a encontrar obstáculos serios durante los años sesenta. 5 A la mitad de esta década, en el patrón de desarrollo rural surge una fractura, que en su momento fue casi imperceptible. Por un lado, la expansión de la tierra cultivable se detiene completamente frente al crecimiento sostenido que había caracterizado dicha expansión hasta 1965. Por otro, la tasa de crecimiento de la producción agrícola, en términos de valor, se reduce también sustancialmente: $2.1 \%$ para el periodo $1966-1975$, en comparación con tasas quinquenales de 4.2 y 8.2 por ciento durante el periodo 1940-1965 (Gómez Oliver, 1978). Otras tendencias igualmente señalan que el proceso de absorción del trabajo agrícola se gestó bajo tensiones y restricciones que iban en aumento. El número de días trabajados por los jornaleros en el sector agrícola disminuyó de manera significativa, de 194 dias en 1950, a 100 en 1960 (Centro de Investigaciones Agrarias, 1974:369). Asimismo, la incorporación de campesinos adicionales al trabajo agrícola se hacía con la reducción del tamaño de las parcelas. Finalmente, el número de campesinos sin tierras progresaba: se estima, aunque los cálculos de este número varían mucho, que en 1970 no poseian tierra de 1 a 3 millones. La cifra más elevada incluye a los campesinos que también eran trabajadores asalariados (Luiselli Fernández, 1977). La absorción de la población en la agricultura, sin olvidar que la información sobre población económicamente activa en este sector debe tomarse con cuidado, creció, a partir de 1960, a un ritmo más lento (véase cuadro 2).

Los eventos posteriores a 1965, han otorgado una perspectiva adecuada al papel que la expansión de la tierra representó como mecanismo para acomodar a la población. La disponibilidad de ésta, era sin duda, un aspecto prioritario para lograr la expansión del campesinado, para quien la tierra es la base material de su existencia y reproducción social. Al agotarse este expendiente, la población rural, que aún va en ascenso, se vio forzada a buscar distintas alternativas al uso de su fuerza laboral. Esta búsqueda, fuera de los asentamientos rura-

5 Las demostraciones (urbanas) estudiantiles de 1968 son consideradas como una prueba significativa de descontento, y como el surgimiento de una actitud más crítica frente a las tendencias y las políticas implementadas anteriormente. 
les de origen campesino, sostuvo y dinamizó patrones de migración del pasado.

Dentro de estos patrones se encuentra la migración temporaria, la cual, en cuanto al sector rural se refiere, parece sustituir a la migración permanente. La migración circular, de carácter estacional (entre regiones agrícolas tanto distantes como cercanas) se convirtió en un fenómeno sobresaliente que llamó la atención de múltiples investigadores en los años setenta. Por un lado, se observa que la migración permanente dirigida a las áreas predominantemente agrícolas del norte del país perdió su anterior dinamismo (Cabrera Acevedo, 1976). Por otro, y con respecto a la migración entre México y Estados Unidos, se encontró que el flujo de trabajo, en especial el vinculado con la actividad agrícola, continuaba con su carácter temporal y circular (CENIET, 1982; Cornelius, 1978).6 Es importante considerar que el trabajo "temporario" también establece vínculos con ciertas actividades en las ciudades, especialmente en lo que toca a la construcción y a ciertos servicios personales. En resumen, la difusión del patrón de migración temporal de la fuerza de trabajo, como patrón demográfico de acomodo, tanto dentro como fuera de la economía nacional, es otro aspecto que añade complejidad a lo que aparentemente era un estilo unidimensional de absorción de la fuerza de trabajo.

La difusión de las modalidades del trabajo temporal así como la realidad de un mercado de trabajo continuo urbano-rural, acentúa las relaciones existentes entre las economias agrícolas, moderna y campesina, en especial a lo largo de la dimensión informal de la economía y la sociedad. Como contrapartida de estos eslabonamientos económicos, aparecen la expansión y permanencia de relaciones y obligaciones sociales (de parentesco, etc.), de carácter campesino que sirvieron a los migrantes rurales para asentarse una y otra vez, tanto fuera como dentro de la economía campesina (el caso ha sido documentado por los migrantes hacía Monterrey y el proceso de retorno en Balán, Browning y Jelin, 1977).

Los acontecimientos anteriores parecen indicar los límites del patrón de acomodo rural que operó entre los años cuarenta y los sesenta. La evolución dual de la agricultura campesina y moderna no lograba acomodar de manera adecuada al número creciente de campesinos que exigía tierra. Asimismo, las dificultades para satisfacer

6 Estas características afectan la estabilidad o inestabilidad del flujo migratorio entre México y Estados Unidos, máxime si este flujo se considera como un mecanismo de absorción de trabajo que parcialmente tiene lugar en las dos economías. 
la demanda de productos agrícolas por parte de la población urbana y la industria se empezaron a manifestar. La autosuficiencia y los superávit en productos de la dieta popular comenzaron a ceder terreno. Durante los años setenta las importaciones de alimentos básicos $\mathrm{y}$ otros productos agrícolas aumentaron enormemente. Las nuevas condiciones del campo mexicano desataron un amplio debate sobre las acciones a seguir para revertir estas tendencias. El punto culminante fue la nueva Ley de Fomento Agropecuario (1980) que enfatiza el objetivo de la productividad para la tierra, incluida la del sector ejidal. (Sobre el debate en torno al agro mexicano véase Schejtman, 1981.)

Las condiciones en el campo sufrieron un cambio en los años sesenta, de igual forma las evoluciones urbana e industrial presentan una nueva fase durante el mismo periodo. El sector formal pierde su capacidad de absorber una oferta de trabajo que crece aceleradamente. En comparación con la dinámica de los años cincuenta, durante los sesenta aparece entre los sectores urbanos -población económicamente activa remunerada-- una tendencia generalizada a la disminución del grado de absorción de la fuerza de trabajo, disminución que resulta más pronunciada en los sectores de servicios (Altimir, 1974: 81 , cuadro 20). El sistema de oportunidades en el trabajo industrialurbano enfrentó, menos satisfactoriamente que en el pasado, la prolongación de tendencias demográficas dinámicas; esto es, un rápido crecimiento de la población y un flujo inmigratorio intenso. 7

Al inicio de los años setenta se hizo evidente que el patrón de crecimiento económico y la modernización urbana (una estrategia de desarrollo peculiar acompañada por acciones sociales gubernamentales muy ambiciosas) no funcionaban adecuadamente para absorber el trabajo. Las condiciones en el mercado de trabajo muestran una amplia segmentación tanto en la agricultura como en la industria y los servicios. Trejo Reyes (1973) estima que en 1965 el 60\% de la fuerza de trabajo ( 8 millones) estaba involucrada en actividades tradicionales, y que la agricultura tradicional comprendía a más de $60 \%$ (5 millones) del total del empleo tradicional. Un grupo de investigadores sobre el problema del empleo estimó que en 1970 cerca de un $30 \%$ de la fuerza de trabajo estaba empleada en el sector tradicional de la economía, y otro $30 \%$ en ocupaciones marginales, principalmente en

7 Aun cuando en el periodo 1960-1970 descendió el peso relativo de los flujos migratorios a las localidades urbanas, la tasa media anual de crecimiento en estas localidades permaneció durante este periodo en el rango de $5.5 \%$ anual (Unikel, 1976). 
Cuadro 4. México: estructura del empleo, 1980

\begin{tabular}{lrrrrr}
\hline & \multicolumn{2}{c}{$\begin{array}{c}\text { Población } \\
\text { activa }\end{array}$} & & & $\begin{array}{c}\text { Ocupaciones } \\
\text { informales }\end{array}$ \\
\cline { 2 - 5 } Sector & Miles & $\%$ & & Miles & $\%^{\mathrm{b}}$ \\
\hline Agricultura & 7025 & 36.1 & & 4670 & 66.5 \\
Minería & 350 & 1.8 & & 39 & 11.1 \\
Manufactura & 3658 & 18.8 & 1090 & 29.8 \\
Construcción & 1362 & 7.0 & & 234 & 17.2 \\
Electricidad & 97 & 0.5 & & - \\
Comercio & 2938 & 15.1 & 1187 & 40.4 \\
Comunicaciones & 877 & 4.5 & & 155 & 17.7 \\
Finanzas & 253 & 1.3 & - & - \\
Otros servicios & 2900 & 14.9 & 759 & 26.2 \\
\hline
\end{tabular}

a Incluye restaurantes y hoteles.

b Porcentaje dentro del sector.

Fuente: Estimaciones (Roitman, 1983).

la agricultura, comercio y servicios diversos; es decir, un total de 7.7 millones de los 13 millones que constituían la población activa.

Cuando se analiza la evolución económica de los años setenta, se estima (Roitman, 1983) que en 1980 las tendencias de absorción laboral no habían cambiado sustancialmente. Las ocupaciones informales de la agricultura todavía representaban el $66.5 \%$ del empleo agrícola; las ocupaciones informales en algunos sectores de servicios fluctuaban entre un 40.4 y un 17.7 por ciento. El empleo informal era sustancial en la manufactura, y representaba casi un 30\%; y en la construcción, un $17.2 \%$ (véase cuadro 4). Estos cálculos, sin embargo, coinciden con estimaciones (PREALC, 1982) que cubren un periodo más largo, de 1950 a 1980 , donde se observa cómo desde 1960 se consolida una clara segmentación del mercado de trabajo en el ámbito urbano. Así el "sector urbano" informal aumenta su participación en los años setenta, de 14.5 a 18.3 por ciento; casi tanto como lo hace durante los años sesenta, de 10.0 a 14.5 por ciento. El incremento del sector informal se llevó a cabo en una época cuando el sector formal urbano también avanzó de manera significativa, del 33.9 al 39.5 por ciento entre 1970 y 1980 (véase cuadro 5).

En suma, pese a que las condiciones sociales y económicas de los años cuarenta y hasta el inicio de los sesenta eran bastante desiguales, los logros en el acomodo demográfico y la absorción de trabajo no generaron conflictos insuperables dentro de la sociedad mexicana. 
Cuadro 5. México: segmentación de la población económicamente activa, $1950,1960,1970$ y $1980(\%)$

\begin{tabular}{lrrrr} 
& 1950 & 1960 & 1970 & 1980 \\
\hline Formal & 21.6 & 32.2 & 33.9 & 39.5 \\
Informal & 9.7 & 10.0 & 14.5 & 18.3 \\
Servicio doméstico & 3.2 & 3.5 & 3.7 & 3.7 \\
$\quad$ Subtotal urbano & 34.5 & 45.7 & 52.1 & 61.5 \\
Moderna & 20.4 & 25.4 & 21.9 & 19.2 \\
Tradicional & 44.0 & 27.6 & 24.9 & 18.4 \\
$\quad$ Subtotal en agricultura & 64.4 & 53.0 & 46.8 & 37.6 \\
Minería & 1.1 & 1.3 & 1.1 & 0.9 \\
Total & 100.0 & 100.0 & 100.0 & 100.0 \\
\hline
\end{tabular}

Fuente: estimaciones (PREALC, 1982: 64, cuadro I-63).

Más aún, el desarrollo de esos años propició un ambiente de expectativas crecientes. Empero, conforme pasó el tiempo, el éxito mismo de las políticas adoptadas abrió el camino a una situación nueva. Una situación que muy esquemáticamente apuntó hacia la consolidación de una sociedad en donde los sectores no incorporados, el tradicional y el informal, se resistieron a ser absorbidos por los sectores mayormente incorporados y transformados: el formal y el moderno.

Así, frente a la creación y la expansión del proletariado industrial, de las clases medias y de un grupo con ingresos elevados y crecientes (sectores con educación. y capacitación profesional y que conforman el clima y la cultura dominantes), se genera una enorme pobreza y marginalización, lo cual dio origen a un drámatico escenario de contrastes. La perpetuación y expansión de redes de carácter familiar, que constituyen importantes instancias de absorción demográfica y laboral se infiltran entre los diversos grupos sociales. Estas redes permitieron a los migrantes rurales encontrar acomodo fuera $o$ dentro de la economía campesina, como en los años sesenta lo documentó el retorno a Cedral de migrantes a Monterrey (Balán, Browning y Jelin, 1977). En los años setenta, los sectores marginales de la ciudad de México aceptan una red social e informal que actúa como sistema efectivo que otorga seguridad y satisfactores a vastos sectores urbanos (Lomnitz, 1975). No se esperaba que la magnitud y las proporciones de este escenario evolucionaran en la forma en que lo hicieron. La estrategia de desarrollo, crecimiento económico rápido 
junto con programas sociales importantes, dio pruebas de no superar, en el contexto de un crecimiento poblacional muy acelerado y de intensos desplazamientos rurales e interurbanos, la acumulación histórica de limitantes económicas y sociales. La absorción del trabajo en los sectores informales y marginales no resultó ser una etapa transitoria, más bien, se convirtió en una característica permanente de la economía y la sociedad. 8

\section{INTENTOS POR ABORDAR EL PROBLEMA DEL EMPLEO}

A mediados de los años sesenta, observadores nacionales y extranjeros aún daban a la economía y sociedad del país "buenas calificaciones". Sin embargo, al final de este mismo periodo, el sistema demoeconómico comenzó a considerarse como problemático. Por un lado, tanto en el campo como en la ciudad, ciertos efectos adversos del patrón de desarrollo adoptado no podían ser ignorados. Por otro, los mecanismos de acomodo económico y social ante las tendencias demográficas parecían enfrentar serios límites.

La expansión de la frontera agrícola, mecanismo fundamental de acomodo rural y de absorción de trabajo, entró en una etapa crítica a mitad de los años sesenta. En el contexto de una frontera agrícola que parecía agotarse, el reparto agrario no fue ya una solución adecuada. Esta actitud indicó que no existía tierra suficiente para garantizar parcelas a todos los campesinos potenciales que aspiraban a contar con ella. Al mismo tiempo se descubrió que la "revolución verde" impactó sólo de manera marginal a la población campesina. De ahí que durante las dos últimas administraciones gubernamentales, (1970-1976 y 1976-1982) se originara una búsqueda de nuevos enfoques y programas en sustitución de la estrategia rural bimodal. Esta búsqueda se orientó hacia el desarrollo rural, con los objetivos de aumentar la producción de granos básicos para la alimentación y de crear empleos. Sobre este último objetivo se observa que, a fin de aumentar la generación de empleo, la inversión rural experimentó en los años setenta un cambio de dirección: de áreas de riego a áreas de temporal. Sin embargo, la información disponible sugiere que el impacto principal de este cambio radica en la creación de empleo temporario, asimismo, que la provisión de empleo permanente es modesta

8 Muchos autores consideran la relación entre el sector formal e informal como un peculiar modus operandi de la escena urbana en los países en desarrollo contemporáneos; un modus operandi que no se encuentra aislado, según Portes, de la evolución del sistema mundial (Portes y Walton, 1981: 84-87). 
si se la compra con la acumulación existente de subocupación rural (Schumacher, 1983).

De igual manera, durante los años sesenta, comenzaron a agotarse en el medio urbano las potencialidades de crecimiento de la etapa "fácil" de industrialización mediante la sustitución de importaciones (Aspra, 1977), a la vez que la elasticidad de absorción de trabajo en relación con el producto disminuía en todos los sectores de la economía (Altimir, 1974:81, cuadro 20). Para los años setenta, el sector industrial presenta un claro descenso en su ritmo de absorción laboral con respecto al pasado. Se ha calculado que la tasa de absorción de trabajo por parte del sector industrial fue del $5 \%$ anual entre 1950 y 1968 , y de $3 \%$ durante el periodo de 1968 a 1978, cuando al enfatizarse la producción de bienes de capital hay un intento de relevo en el motor de crecimiento de la economía. Frente a ello, la expansión del sector público, que caracterizó el inicio de los años setenta, suplió en alguna medida la pérdida de capacidad industrial, para convertirse posteriormente en un factor importante de absorción laboral. Se ha estimado que bajo la administración de Luis Echeverría (1970-1976), los empleados gubernamentales de cerca de medio millón aumentaron a más de un millón. Debe observarse que, en general, los empleos gubernamentales se inscriben en los sectores incorporados o formales.

Dentro de esta línea de modernización y transformación del país, la decisión de explotar rápidamente las ventajas de la extracción acelerada del petróleo en los años setenta no es ajena a la problemática del empleo. En este periodo, el país vivió una expansión económica acelerada, con una tasa de crecimiento del PIB por encima del $8 \%$ entre 1978 y 1981 . Se trató, tal vez, del intento más audaz por lograr una completa modernización de la sociedad y de la economía mexicana. Las promesas eran grandes al considerar que con la desaparición de la restricción financiera del horizonte, era posible, por fin, subsanar el cúmulo de antiguas carencias económicas y sociales, y con ello lograr la transformación del país. El plan de desarrollo industrial 1979-1982 englobó una estrategia de empleo que se apoyaba en la aceleración de la tasa de absorción formal de la fuerza de trabajo; se proyectó que los segmentos laborales remunerados aumentaran a una tasa del $5.1 \%$ anual durante el periodo de 1980 a 1985.9

La bonanza de la economía trajo consigo aumentos sustanciales en la creación de empleos. En el periodo de 1978 a 1981 éstos crecie-

9 Se hicieron intentos por desarrollar un programa nacional de empleo, sin embargo, las propuestas (1979) no alcanzaron una formación política definitiva. 
ron a tasas más elevadas que la tasa de aumento de oferta de fuerza de trabajo. Se suponia que si la economía se mantenía en ascenso entre el 8 y 10 por ciento, eventualmente se tendría éxito en absorber el trabajo no utilizado. Sin embargo, en 1982 el crecimiento del país tuvo una interrupción y la economía entró en una recesión profunda. En los años setenta, la crisis cambió la perspectiva de los logros obtenidos. Es evidente que éstos fueron de poca duración. Además, la experiencia de un crecimiento muy acelerado se asoció con algunas paradojas aparentes. Había escasez de fuerza de trabajo en una situación que se caracterizó por su abundancia. Aparecieron síntomas de desajustes y cuellos de botella en el proceso de absorción del trabajo; el calificado se volvió escaso y caro, mientras que el no calificado se mantuvo abundante y barato.

Es muy pronto aún para evaluar el impacto de largo plazo del "boom" económico que propició la acelerada explotación del petróleo. De cualquier modo, todo indica que la consolidación de los sectores informales que se delinean como una característica del escenario urbano en los años sesenta y setenta (Lomnitz, 1975) continúa su avance. Algunos de los patrones de acomodo de los marginados urbanos empiezan a llegar a su límite. La segmentación del mercado de trabajo y la polarización de la sociedad mexicana se convierten en una condición estructural que refleja las tensiones del sistema demograficoeconómico. Es indudable que los grupos medios y los trabajadores sindicalizados se han incrementado, y con ello ganado fuerza y poder. Sin embargo, los sectores informales y marginales, integrados parcialmente a las instituciones nacionales, cobraron dinamismo. Actualmente compiten intensamente con el moderno y formal. Estos sectores, que en el pasado amortiguaron un patrón de desarrollo dual y desigual, pero parcialmente exitoso, podrían amenazar, por la sola expansión de sus números, al mismo patrón de desarrollo que los generó.

\section{¿Signos de CAMbio EN El FUTURo?}

Las proyecciones de las tendencias demográficas, aunque de forma relativa, ofrecen apoyo a una perspectiva menos pesimista. Al principio de los años setenta se inició un descenso en el crecimiento de la población. 10 Sin embargo, las implicaciones en la fuerza de trabajo

10 El crecimiento de la población en México era del $3.4 \%$ al inicio de los años setenta, y de $2.7 \%$ al inicio de los años ochenta (Consejo Nacional de Población, 1982). 
de este decremento no podrán manifestarse, sino hasta cerca del año 2000. Los cálculos disponibles muestran que la población en edad de trabajar se expandirá de 36 a 67 millones en el periodo que comprende los años 1980 al 2000 (Secretaría de Programación y Presupuesto, 1983). En términos de población activa, se calcula que ésta se duplicará en el mismo periodo, de 20 a 35 o 40 millones, por lo cual se requiere crear alrededor de un millón de nuevos empleos cada año, hasta el final del siglo. El volumen de la futura población activa está sujeta a un amplio margen de variación, puesto que el grado de incorporación de la mujer al mercado de trabajo representa un factor decisivo en los futuros números de la fuerza de trabajo. Su ritmo de incorporación podría acelerarse rápidamente o estancarse. Lo primero parece más factible que lo segundo, ya que la experiencia en México indica una cada vez mayor participación femenina en los mercados laborales.

No existe duda alguna de que el país precisa de un crecimiento económico acelerado para tener capacidad de crear una demanda laboral proporcional al número que ingresará al mercado de trabajo. Sin embargo, las perspectivas que se preveen a mediano plazo en la economía mundial, así como la crisis de México, han cuestionado la estrategia petrolera considerada como motor de un crecimiento económico que pudiera dar solución a la demanda de empleos productivos, lo cual se convertiria en un proceso del tipo "trickle-down". De hecho, la estrategia de desarrollo que a partir de los años cuarenta se implementó, termina por ser complementada con programas específicos de empleo. El programa de construcción de "caminos alimentadores" para dar ocupación a la mano de obra rural, que se realizó a principios de los años setenta, es tal vez la primera de las acciones en este campo.

Dichas acciones se han continuado y es de preveer que en el futuro cercano habrá presiones para que se amplien y no desaparezcan. Aun cuando se estructure una estrategia de desarrollo rural que tenga éxito en áreas de temporal, no es factible esperar un cambio rápido en la situación laboral que prevalece en el campo mexicano. La experiencia del SAM es ilustrativa. Se dedicaron muchos recursos y se obtuvieron resultados en la dirección de una mayor productividad y de un incremento de empleos, pese a ello la situación en el campo no se ha podido revertir. Los aumentos de productividad en la agricultura de temporal indican que lograr este proceso de reversión es extremadamente lento (Schumacher, 1983).

La transformación de las condiciones que imperan en el campo es todavía un proceso de largo alcance para las tendencias de la absor- 
ción futura del trabajo. La población rural aún es muy numerosa: en 1980 , alrededor de 25 millones de personas vivían en lugares con menos de 2500 habitantes. Actualmente la población rural se encuentra en ascenso. Asimismo, es probable que el número de campesinos y trabajadores agricolas sin tierras se eleve. El número de unidades agrícolas en estado de descomposición y desorganización es considerable. Algunas estimaciones señalan que el número de estas unidades ascendía en 1970 a 2.5 millones, lo que representaba el $78 \%$ del total (Luiselli Fernández, 1977).

Cuando se confrontan las realidades del campo, hay quienes argumentan que la economía campesina debe ser revitalizada y recrdenada; otros ven el declinar de la economía campesina como inevitable, pese a que no es claro lo que sucederá con sus miembros. (Al respecto se ha producido un amplio debate; sobre el mismo véase Schejtman, 1981.) En cualquier caso, es seguro que la evolución de las condiciones del campo pesarán con fuerza sobre el mercado de trabajo urbano. Parece difícil suponer que el sector rural deje de dirigir permanentemente su población a las ciudades, en especial su fuerza de trabajo excedente, o en los periodos en que ésta se encuentra desocupada o subutilizada.

Ante estas circunstancias, el sector urbanoindustrial está llamado a ser el medio en donde se da el proceso de la absorción del trabajo. La estrategia de la administración López Portillo (1976-1982) fue revigorizar el desarrollo industrial, a la vez que se promovieron centros urbanos alternos - viejos y nuevos- al de la ciudad de México. Uno de los ejes de esta estrategia era la creación de puertos industriales en áreas costeras, tanto en el golfo como en el Pacífico. No obstante la concreción de avances importantes en el desarrollo industrial y la desconcentración urbana, las nuevas (post, 1982) circunstancias económicas -ajustes de programas y restricciones financieras- han detenido las inversiones o las han llevado a un ritmo extremadamente lento. Dentro de este cuadro de recesión, se calcula que en 1984 el sector moderno podría proporcionar cerca de 100000 empleos -de los 800000 que son necesarios-, la agricultura 100000 más y otros tantos el sector informal (Políticas de empleo, 1984). De lo anterior se desprende que sería de la competencia de los distintos programas de empleo, absorber el resto de ingresos al mercado de trabajo.

Si la actual situación de lento crecimiento se prolonga, la sociedad mexicana enfrentará el dilema de institucionalizar programas de empleo que después resultarán difíciles de desmantelar. No tenerlos sería socialmente inaceptable; pero perpetuarlos puede significar la introducción de rigidez en el mercado de trabajo. Es necesario aña- 
dir que en las circunstancias actuales donde existe una abundante oferta laboral, hay presiones a lo largo de todo el sistema económico, para reducir los salarios y otras prestaciones al trabajo, con el fin de absorber o facilitar la incorporación de segmentos adicionales e importantes de mano de obra al mercado de trabajo. En otras palabras, es claro, que los problemas de absorción laboral deberán considerar, en mayor medida que en el pasado, el debate de la política económica y social, si se aspira a encontrar patrones más aceptables de incorporación económica de la creciente fuerza de trabajo del país.

\section{Bibliografía}

AltimiR, Óscar, "La medición de la población económicamente activa de México, 1950-1970", Demografía y Economia, 8, núm. 1,1974

ASPRA, L. Antonio, "Import Substitution in Mexico: Past and Present", World Development (enero-febrero), 1977.

BALÁN, Jorge, Browning Harley L. y Jelin Elizabeth, El hombre en una sociedad en desarrollo; movilidad geográfica y social en Monterrey, México, Fondo de Cultura Económica, 1977.

CABrera Acevedo, Gustavo, "Población, migración y fuerza de trabajo", en Mercados regionales de trabajo, México, Instituto Nacional de Estudios del Trabajo/Naciones Unidas, 1976.

CENTRO DE ESTUdios ECONÓMICOS Y DEMOGRÁfICOS, Dinámica de la población de México, México, El Colegio de México, 1970.

CENTRO DE INVESTIGACIONES AGRARIAS, Estructura agraria y desarrollo agricola en México, México, Fondo de Cultura Económica, 1974.

CENTRO NACIONAL DE INFORMACIÓN Y ESTADÍSTICAS DEL TRABA JO, Los trabajadores mexicanos en Estados Unidos, México, 1982.

CEPAL, Economia campesina y agricultura empresarial, México, Siglo XXI, 1982

Consejo Nacional de Población, México Demográfico. Breviario, 1980-1981, México, 1982.

CORNELIUS, Wayne A., "Mexican migration to the United States: Causes, consequences and U.S. responses". Center for International Studies, Massachusetts Institute of Technology, junio, 1978.

GARCÍA, Brígida, "La participación de la población en la actividad económica", Demografía y Economia, 9, núm. 1, 1975.

Gómez OLIVER, Luis, "Crisis agrícola, crisis de los campesinos", Comercio Exterior, 28, núm. 6, junio, 1978. 
GRUPO DE EstUdio DEL PROBleMA DEL EMPLEO, El problema ocupacional en México. Magnitud y recomendaciones (versión para discusión).

HEWITT DE ALCÁNTARA, Cynthia, La modernización de la agricultura mexicana, 1940-1970, México, Siglo XXI, 1980, segunda edición.

Lomnitz, Larissa, Cómo sobreviven los marginados, México, Siglo XXI, Editores, 1975.

LUISELLI FERNÁNDEZ, Cassio, "La crisis agropecuaria y la política demográfica", en Opciones de política económica en México, México, Ed. Tecnos, 1977.

Morelos, José B., La situación del empleo y subempleo en México (mimeo), 1974.

"POLÍTICAS DE EMPLEO", El mercado de valores, XLIV, núm. 10, 5 de marzo, 1984.

PORTES, Alejandro, "Unequal exchange and the urban informal sector", en Labor, Class, and the International System, Alejandro Portes y John Walton, Nueva York, Academic Press, 1981.

PREALC, Mercado de trabajo en cifras, 1950-1980, PREALC-OIT, 1982.

ROITMAN, Benito, El empleo en México hacia 1983: proyecciones y perspectivas (mimeo), septiembre, 1983.

SCH JTMAN, Alejandro, "El agro mexicano y sus intérpretes", Nexos, núm. 39, marzo, 1981.

SCHUMACHER, August, "Agricultural Development and Rural Employment: A Mexican Dilema", en The Border that Joins, Peter G. Grown and Henry Shue (eds.), Totowa, N.J.: Rowman y Littlefield, 1983.

Secretaría de Programación y Presupuesto, Instituto Nacional de Estadística, Geografía e Informática, Consejo Nacional de Población y Centro Latinoamericano de Demografía, México. Estimaciones y proyecciones de población, 1950-2000, México, octubre, 1983.

TREjo REYES, Saúl, Industrialización y empleo en México. México, Fondo de Cultura Económica, 1973.

UNIKEL, Luis, El desarrollo urbano de México: diagnóstico e implicaciones futuras, México, El Colegio de México, 1976.

URQUIDI, Víctor L., "Empleo y explosión demográfica", Demografia y Economia, 8, núm. 2, 1974.

VAN GINNEKEN, Wouter, Socio-economic Groups and Income Distribution in Mexico, Londres, Croom Helm (ILO), 1980. 